\title{
Rapid cell corpse clearance by stabilin-2, a membrane phosphatidylserine receptor
}

\author{
S-Y Park ${ }^{1,2,3}$, M-Y Jung ${ }^{1,3}$, H-J Kim ${ }^{1}$, S-J Lee ${ }^{1}$, S-Y Kim ${ }^{1}$, B-H Lee ${ }^{1}$, T-H Kwon ${ }^{1}$, R-W Park ${ }^{1}$ and I-S Kim ${ }^{\star, 1}$
}

Rapid phagocytic clearance of apoptotic cells is crucial for the prevention of both inflammation and autoimmune responses. Phosphatidylserine (PS) at the external surface of the plasma membrane has been proposed to function as a general 'eat me' signal for apoptotic cells. Although several soluble bridging molecules have been suggested for the recognition of PS, the PS-specific membrane receptor that binds directly to the exposed PS and provides a tickling signal has yet to be definitively identified. In this study, we provide evidence that stabilin-2 is a novel PS receptor, which performs a key function in the rapid clearance of cell corpses. It recognizes PS on aged red blood cells and apoptotic cells, and mediates their engulfment. The downregulation of stabilin-2 expression in macrophages significantly inhibits phagocytosis, and anti-stabilin-2 monoclonal antibody provokes the release of the anti-inflammatory cytokine, transforming growth factor- $\beta$. Furthermore, the results of timelapse video analyses indicate that stabilin-2 performs a crucial function in the rapid clearance of aged and apoptotic cells. These data indicate that stabilin-2 is the first of the membrane PS receptors to provide tethering and tickling signals, and may also be involved in the resolution of inflammation and the prevention of autoimmunity.

Cell Death and Differentiation (2008) 15, 192-201; doi:10.1038/sj.cdd.4402242; published online 26 October 2007

Apoptosis is an essential physiological process for embryonic morphogenesis and for the maintenance of homeostasis in adult multicellular organisms. ${ }^{1}$ Rapid and efficient cell corpse clearance is crucial to the protection of normal healthy cells from harmful contents and the debris of dying cells, and such clearance also reduces the tissue damage resulting from inappropriate inflammation or autoimmune responses. ${ }^{2}$ Clearance is generally achieved via the activity of professional phagocytes (macrophages and immature dendritic cells) and neighboring cells (fibroblasts, endothelial, and epithelial cells). The central element in the recognition process is the cell surface presentation of adhesive engulfment ligands (also referred to as 'eat me' signals) displayed by apoptotic cells. .,4 $^{3,4}$ Although little remains known regarding the molecular identities of engulfment ligands, the best-characterized marker of apoptotic cells is phosphatidylserine (PS) exposure, which has been associated with losses in phospholipid asymmetry.

Several classes of receptors have been implicated in the recognition of apoptotic cells, possibly via direct binding to the exposed PS or indirect binding through bridging molecules. ${ }^{3}$ These receptors include the phosphatidylserine receptor (PSR), ${ }^{5}$ C. elegans CED $-1,{ }^{6}$ CD $14,{ }^{7}$ the $\alpha \mathrm{v} \beta 3$ and $\alpha \mathrm{v} \beta 5$ integrins, ${ }^{8}$ and the receptor tyrosine kinase Mer. ${ }^{9}$ In addition, several scavenger receptors have been theorized to mediate the binding and uptake of aged/apoptotic cells, including LOX-1, ${ }^{10}$ CD36, ${ }^{11}$ Drosophila Croquemort, ${ }^{12}$ and low-density lipoprotein (LDL)-receptor-related protein (LRP1, also referred to as CD91). ${ }^{13,14}$ These receptors may function in parallel or redundant phagocytic systems, as the blocking of any receptor impairs apoptotic cell engulfment only partially. ${ }^{15}$ Although these receptors have been implicated in the engulfment of aged/apoptotic cells, no evidence has yet been presented to show that any of these receptors can directly recognize PS. Although the above-mentioned bridging molecules have been demonstrated to recognize PS, their activity is insufficient to explain all of the observed responses to PS, as well as the attendant anti-inflammatory and anti-immunogenic effects. PSR was originally shown to be a specific PS receptor, but now it appears not to perform this function. Therefore, a membrane receptor for PS remains to be identified. $^{16}$

Stabilin-2 is a large multifunctional receptor, which harbors seven FAS1 domains, four EGF-like repeats, and a link domain. Stabilin-2 was initially identified as the endocytic hyaluronan receptor on rat liver sinusoidal endothelial cells ${ }^{17,18}$ and has also been reported to function as a scavenger receptor, which effects the endocytosis of modified LDL and glycation end products. ${ }^{19,20}$ Stabilin-2 also has been shown to bind to Gram-negative and Gram-positive bacteria, thereby suggesting the potential involvement of stabilin-2 in the immune response. It is expressed at high levels in the sinusoidal endothelial cells of the spleen, lymph nodes, and liver. ${ }^{18,21}$ In this study, we present evidence that stabilin-2 is

\footnotetext{
${ }^{1}$ Department of Biochemistry and Cell Biology, Cell and Matrix Research Institute, School of Medicine, Kyungpook National University, Daegu, Korea and ${ }^{2}$ Department of Biochemistry, School of Medicine, Dongguk University, Kyungju, Korea

${ }^{*}$ Corresponding author: I-S Kim, Department of Biochemistry and Cell Biology, Cell and Matrix Research Institute, School of Medicine, Kyungpook National University, Daegu 700-422, Korea. Tel: + 8253420 4821; Fax: + 8253422 1466; E-mail: iskim @knu.ac.kr

${ }^{3}$ These authors contributed equally to this work.

Keywords: phagocytosis; phosphatidylserine; macrophages; stabilin-2; FEEL-2; HARE

Abbreviations: PS, phosphatidylserine; RBCs, red blood cells; TGF- $\beta$, transforming growth factor- $\beta$; PSR, phosphatidylserine receptor; LDL, low-density lipoprotein; PC, phosphatidylcholine; NBD, 7-nitrobenz-2-oxa-1,3-diazole; HMDMs, human monocyte-derived macrophages; mAb, monoclonal antibody; shRNA, short hairpin RNA; PMA, phorbol myristate acetate; FITC, fluorecein-5-isothiocyanate.

Received 26.2.07; revised 19.7.07; accepted 12.9.07; Edited by M Piacentini; published online 26.10.07
} 
also expressed in human monocyte-derived macrophages (HMDMs). These findings compelled us to speculate that stabilin-2 may participate in the recognition and engulfment of apoptotic and aged cells in macrophages. Accordingly, the potential for stabilin-2 to function as a PS receptor in the clearance of aged and apoptotic cells is addressed and supported by the evidence reported herein.

\section{Results}

Stabilin-2 performs a crucial function in the rapid engulfment of aged RBCs. Stabilin- 2 has been identified as a multifunctional scavenger receptor that endocytoses modified LDL and glycation end products and also evidences Gram-negative and Gram-positive bacterial-binding activities. $^{19,20}$ However, the possibility that stabilin-2 might participate in the phagocytosis of PS-exposed cell corpses remained an open question until now. In an effort to assess the potential involvement of stabilin-2 in the recognition and engulfing of apoptotic and aged cells, stabilin-2 was stably transfected into mouse fibroblast $L$ cells, which evidence no phagocytic activity under normal conditions. L cells have been shown not to express any cadherins ${ }^{22}$ and they have the advantage of being able to distinguish stabilin-2-specific binding from other interactions, because they do not interact significantly with any of the normal or apoptotic cells employed in this study. Through selection in G418, four lines were acquired that consistently expressed stabilin-2 on the cell surface (Figure 1a). We selected aged red blood cells (RBCs) as target cells, because they do not bind to phagocytes without apoptotic signals, and allow for distinction between binding and engulfment via hypotonic lysis. ${ }^{23}$ Stabilin-2 stably expressing $L$ cells (L/Stab-2) were shown to bind to and engulf aged RBCs $(79.4 \pm 5.9$ and $41.4 \pm 4.8$, respectively) to a significant degree, whereas $L$ cells transfected with the empty vector (L/Mock) evidenced little binding to and absolutely no phagocytosis of aged RBCs $(13.4 \pm 4.5$ and $2.0 \pm 0.7$, respectively) (Figures $1 \mathrm{~b}$ and $\mathrm{c}$ ). The interaction between RBCs and L/Stab-2 cells was specific to aged cells, as the normal RBCs did not interact with the L/Stab-2 cells in this assay (Figure 1c). To verify the engulfment of aged RBCs, the phagocytosis assay was modified via the pre-labeling of the target cells with phosphatidylcholine (PC)-7-nitrobenz-2-oxa-1,3-diazole (NBD). Distinctly labeled target cells (green) were detected within the L/Stab-2 cells (red) (Figure 1d). Pretreatment with a monoclonal anti-stabilin-2 antibody (mAb 5G3) before exposure to aged RBCs resulted in a substantial reduction in the binding and uptake of aged RBCs (from 78.8 \pm 11.1 and $42.1 \pm 3.9$ to $15.8 \pm 5.3$ and $17.7 \pm 6.5$, respectively) (Figure 1e). This antibody is specific to stabilin-2 and does not crossreact with orthologous and paralogous proteins (Supplementary Figure 1 is available online at http://

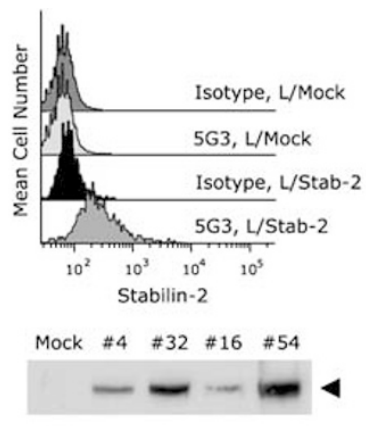

b

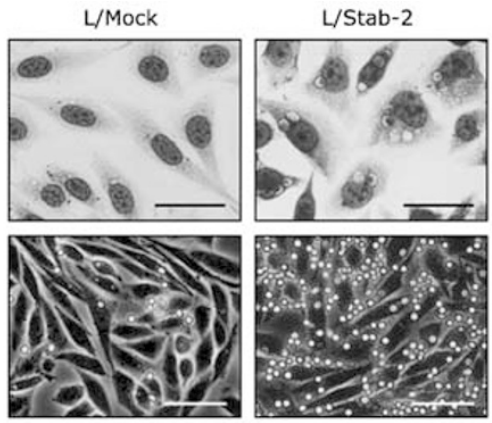

C

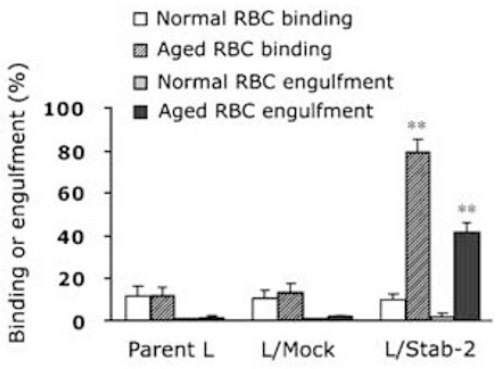

d

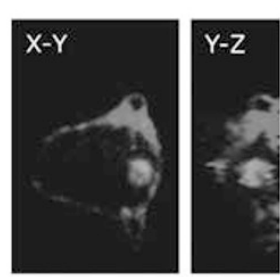

e

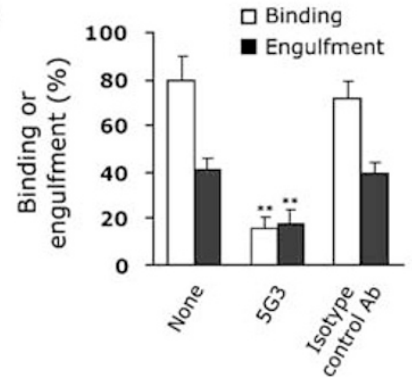

f

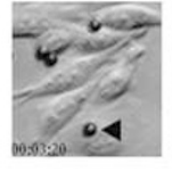

g

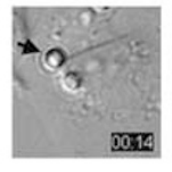

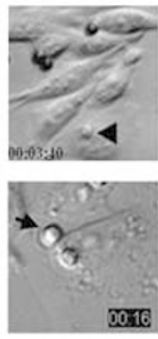
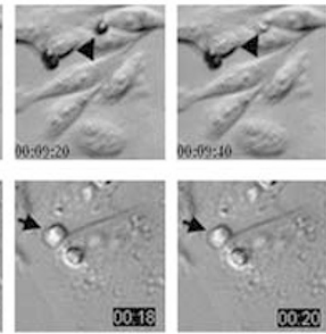

Figure 1 Binding and engulfment of aged RBCs by $L$ cells expressing stabilin-2-Myc (L/Stab-2). (a) (Top) Flow cytometry detection of stabilin-2 expression on $L$ cells stably transfected with stabilin-2 cDNA (L/Stab-2) or empty vector (L/Mock). (Bottom) Immunoblot analysis of four L/Stab-2 clones. Stabilin-2-Myc expression was evaluated via immunoblotting with antibody to Myc (anti-Myc). The arrowhead indicates stabilin-2 protein. (b) Representative images of binding (Bottom) and engulfment (Top) of aged RBCs in L/Stab-2 and L/Mock cells. Scale bar, $50 \mu \mathrm{m}$. (c) Microscopic quantitation of binding and engulfment of aged RBCs in parent L, L/Mock, and L/Stab-2 cells. The results are expressed as the means \pm S.D. from at least three experiments. ANOVA: ${ }^{* *} P<0.01$. (d) L/Stab-2 cells were incubated with PC-NBD-labeled aged RBCs (green) for 30 min at $37^{\circ} \mathrm{C}$, fixed with $3.7 \%$ formaldehyde, and stained with anti-F-actin antibody (red). L/Stab-2 cells were imaged with a spinning disk confocal microscope. $X-Y$ and $Y-Z$ sections are shown. (e) The inhibition of aged RBCs binding $(\square)$ and engulfment ( $\square$ ) by pretreatment with a monoclonal anti-stabilin-2 antibody (5G3). The percentages of cells binding to or engulfing aged RBC were determined. The results are expressed as the means \pm S.D. from at least three experiments. ANOVA: ${ }^{* *} P<0.01$. (f) DIC images from Supplementary Movie 1 show the engulfment of aged RBCs (black arrowheads) by L/Stab-2 cells. (g) Individual frames from Supplementary Movie 2, showing the engulfment of aged RBC (black arrows) by L/Stab-2 cells. The images were acquired every $2 \mathrm{~s}$ 
a

840 (shRNA1)

- AAAAC AAACTGGAAT GCAAATGCCT TCCCAATTAC CGAGGGGATG gCAAATACTG CGACCCCATC AATCCATGTT TACGAAAAAT CTGCCACCCT CATGCTCATT GTACGTACCT 1324 (shRNA2)

GAGGA AAATGGCAAG GAAGGCTGAC CTCTTTCATC TCACTCCTAG ACAAAGCTTA TGCCTGGCCA CTGAGTAAGC TGGGACCCTT CACGGTGCTG TTACCTACAG ACAAGGGACT 3174 (ShRNA3)

TGITT GTCAAGAGGG CTATGAAGGA GATGGCTTTC TGTGCTATGG AAACGCAGCA GTGGATTG CATTTCTCTC CGAGGCAGCT ATATTTAACC GATGGATAAA TAATG......

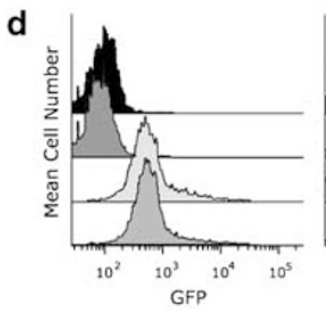

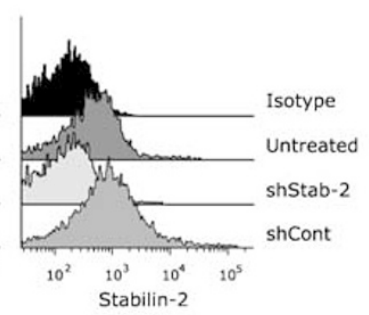

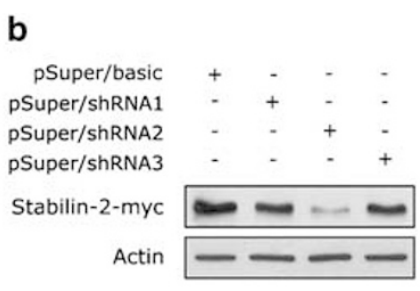

C

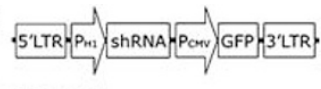

shStab-2

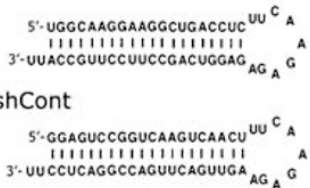

e

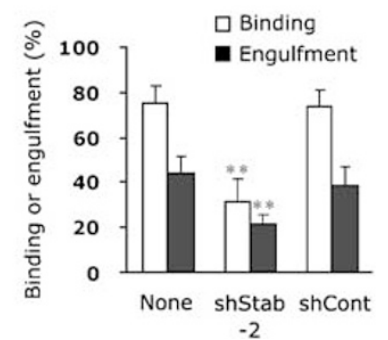

Figure 2 Stabilin-2 knockdown inhibits the binding and engulfment of aged RBCs. (a) The location of shRNAs in stabilin-2 mRNA. Three shRNA candidates were designed and cloned into pSuper vector. (b) Confirmation of the shRNA-induced suppression of stabilin-2 expression. CHO-K1 cells were cotransfected with pSuper/basic, pSuper/ shRNA1, pSuper/shRNA2, or pSuper/shRNA3, coupled with a stabilin-2-expressing vector. Stabilin-2 expression was evaluated via immunoblotting with antibody to Myc (antiMyc). (c) Schematic drawing of lentiviral vector for knocking down stabilin-2 (shStab-2) and scrambled shRNA vector (shCont). (d) shRNA-induced the downregulation of stabilin-2 expression in L/Stab-2 cells. The cells were infected with a lentivirus containing either stabilin-2 shRNA (shStab-2) or scrambled control shRNA (shCont). At $72 \mathrm{~h}$ after infection, the expression of GFP protein (left panel) and the suppression of stabilin-2 expression (right panel) were confirmed via flow cytometry. (e) The inhibition of aged RBC binding $(\square)$ and engulfment ( $\mathbf{\square}$ ) by stabilin-2 shRNA (shStab-2) pretreatment. The percentages of cells binding or engulfing aged RBC were determined. The results are expressed as the means \pm S.D. from at least three experiments. ANOVA: ${ }^{* *} P<0.01$

www.nature.com/cdd/index.html). We also evaluated the interaction occurring between L/Stab-2 cells and aged RBCs via time-lapse video microscopy. Aged RBCs were quickly engulfed by L/Stab-2 cells (Figure $1 f$ and Supplementary Movie 1). To further investigate the velocity of uptake, the images were analyzed at shorter intervals. We observed that the uptake of tethered RBCs occurred within 2-4 s (Figure 1g and Supplementary Movie 2).

In an effort to further determine the potential involvement of stabilin-2 in cell corpse removal, we designed three short hairpin RNAs (shRNA)-targeting stabilin-2 (Figure 2a) and cloned them into the pSuper vector. The suppression of stabilin-2 expression was evaluated in $\mathrm{CHO}-\mathrm{K} 1$ cells via the co-transfection of the shRNAs along with a stabilin-2expressing vector. The expression of stabilin-2 was suppressed profoundly by shRNA2, but only marginally by shRNA1 and shRNA3 (Figure 2b). To suppress effectively the expression of stabilin-2, lentiviruses encoding for stabilin2 shRNA2 (shStab-2) or its scrambled shRNA (shCont) along with green fluorescent protein (GFP) were prepared (Figure 2c). Under similar transfection efficiency conditions, shRNA against stabilin-2, but not control shRNA, downregulated the expression of the stabilin-2 protein (Figure $2 \mathrm{~d}$ ) and significantly reduced the binding and uptake of aged RBCs (from $75.6 \pm 7.3$ and $44.2 \pm 7.4$ to $31.4 \pm 10.1$ and $21.6 \pm 4.3$, respectively) (Figure $2 \mathrm{e}$ ). Collectively, our results indicate that stabilin-2 specifically mediates the binding and engulfment of aged RBCs.

Stabilin-2 recognizes PS and engulfs aged RBCs in a PS-specific manner. Although the molecular identities of engulfment ligands remain largely unknown, PS externalization has been identified as a hallmark of the earliest stages of the apoptotic process. ${ }^{24,25}$ The exposure of PS to the surface of aged RBCs increased in a timedependent manner (Figure 3a, left panel). These changes were accompanied by an increase in binding to L/Stab-2 cells (Figure 3a, right panel), thereby indicating that stabilin-2 may recognize PS on the surfaces of aged RBCs. We then evaluated the affinity of fluorescence-labeled liposomes harboring a variety of phospholipids to monolayers of $\mathrm{L} /$ Stab-2 cells. As is shown in Figure 3b, PS liposomes bound to L/Stab-2 cells to a significant degree, but other phospholipid liposomes did not. None of the phospholipid liposomes bound to the L/Mock cells to any significant extent. We evaluated the engulfment of aged RBCs by L/Stab- 2 cells in the presence of PS liposomes, using PC liposomes as a negative control. The PS liposomes effected a dosedependent inhibition of engulfment of aged RBCs by L/Stab-2 cells, whereas the PC liposomes exerted no such effects (Figure $3 c$ ). The inhibition of aged RBC binding and uptake was restricted to the PS liposomes, and other anionic and neutral phospholipids exerted no detectable effects (Figure $3 d$ ). To confirm the specific recognition of PS, we assessed the inhibitory action of a structural analogue of PS using phospho-L-serine (PLS), with phospho-D-serine (PDS) employed as a negative control. Similarly, binding and uptake were inhibited by PLS, but not by PDS (Figure 3e). These findings show that L/Stab-2 cells do not simply interact with aged RBCs through their negative charge, but that they recognize PS stereospecifically on the surfaces of the aged cells.

Stabilin-2 engulfs apoptotic cells via the recognition of PS on the surfaces of apoptotic cells. We conducted phagocytosis assays using apoptotic cells as target cells. Jurkat T and U937 cells were induced to undergo apoptosis 

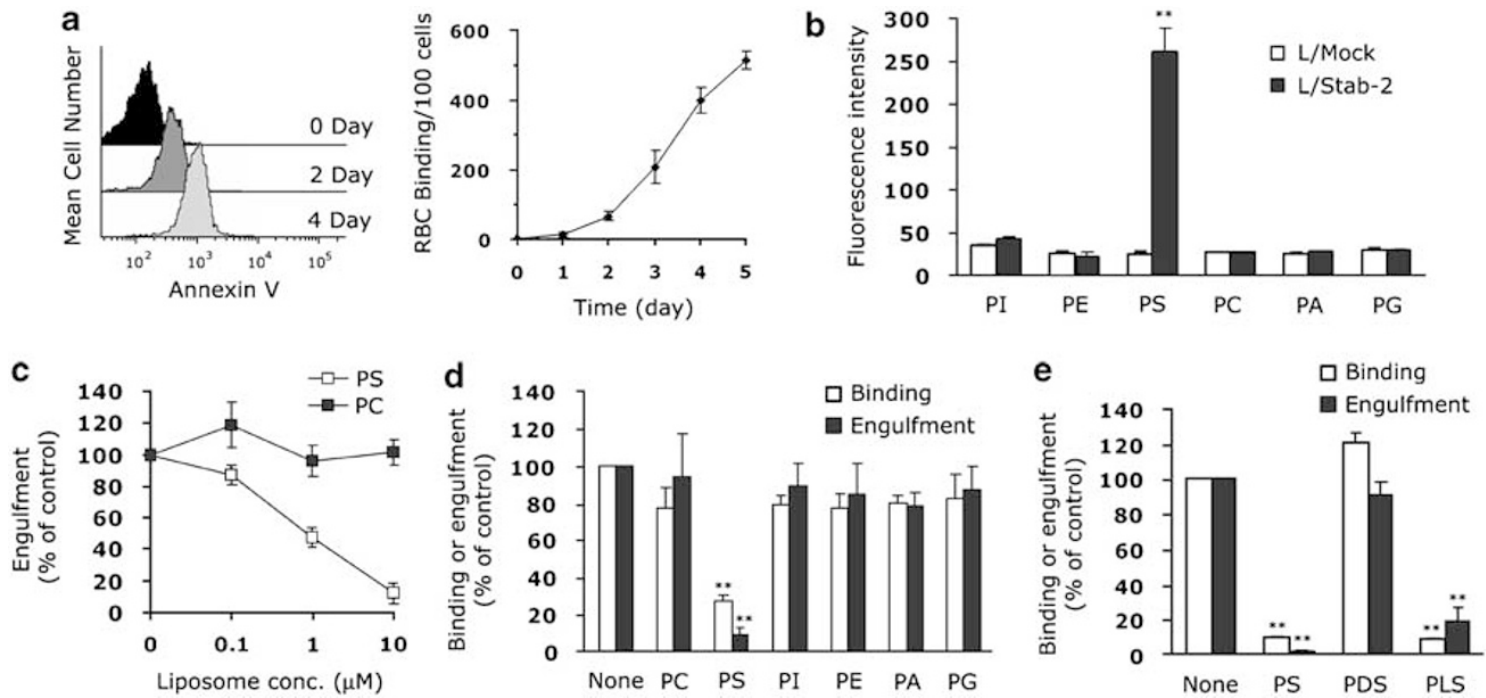

Figure 3 Stabilin-2 mediates the binding and engulfment of aged RBCs in a PS-specific manner. (a) RBCs were induced to undergo aging for various time points (left panel), and the binding of RBC to L/Stab-2 cells was determined (right panel). (b) Binding of fluorescence-labeled liposomes composed of various phospholipids in L/Mock $(\square)$ or L/Stab-2 $(\boldsymbol{\square})$ cells. The results were expressed as the means \pm S.D. from at least three experiments. ANOVA: ${ }^{*} P<0.01$. (c) Engulfment of aged RBCs by L/Stab-2 cells in the presence of PS $(\square)$ or PC liposomes $(\mathbf{\square})$. The results are expressed as percentages of cells binding to or engulfing aged RBCs when compared with untreated cells. The results are expressed as the means \pm S.D. from at least three experiments. (d and e) Binding ( $\square$ ) and engulfment ( $\mathbf{\square}$ ) of aged RBCs by L/Stab-2 cells in the presence of various phospholipids $(10 \mu \mathrm{M})(\mathbf{d})$ or its structural analogues $(10 \mu \mathrm{M})(\mathbf{e})$. PC, phosphatidylcholine; PE, phosphatidylethanolamine; PI, phosphatidylinositol; PG, phosaphatidylglycerol; PA, phosphatidic acid; PLS, phospho-L-serine; PDS, phospho-D-serine. The results are expressed as the percentages of cells that bind to or engulf aged RBC when compared with untreated cells. The results are expressed as the means \pm S.D. from at least three experiments. ANOVA: ${ }^{* *} P<0.01$

via treatment with anti-Fas antibody or etoposide, respectively. Apoptosis was quantified via the binding of fluorecein-5-isothiocyanate (FITC)-labeled annexin $V$ to externalized PS, and was confirmed by staining with propidium iodide $(\mathrm{PI})$, which cannot permeate the intact plasma membranes of apoptotic cells (Figure 4a). As had been expected, the L/Stab-2 cells engulfed the apoptotic cells (Figures $4 b$ and $c$ ), and this uptake was inhibited significantly by mAb $5 \mathrm{G} 3$ and the PS liposome, thereby suggesting that the PS-specific engulfment by stabilin-2 did not depend on the type of cells or the initiating signals (Figure 4c). We also evaluated the engulfment of apoptotic cells by L/Stab-2 cells using time-lapse video microscopy. Phagocytosis was delayed until $\sim 5 \mathrm{~min}$ (Figure $4 \mathrm{~d}$ and Supplementary Movie 3), and the elongation of ingestion time may be resultant from the different size of the target cells. We then utilized Jurkat T cells coated with PS in order to directly assess the role of stabilin-2 as a PS receptor involved in the binding and engulfment of apoptotic cells. As is shown in Figure 5a, these cells acquired the ability to bind to annexin V. Viable Jurkat $T$ cells coated with PS in the outer leaflet were engulfed by L/Stab-2 cells, in a manner comparable to that of apoptotic cells $(31.2 \pm 5.2$ and $35.7 \pm 10.2$, respectively) (Figure $5 b$ ). Viable cells coated with PC were not engulfed (Figure $5 b$ ). When the PS-treated viable cells were cultured for $6 \mathrm{~h}$ after the PS liposomes were washed away, they no longer bound to annexin V, and were therefore not engulfed (Figures $5 c$ and $d$ ), thereby indicating that PS liposome treatment did not induce the apoptosis of viable cells. These results indicate that $P S$ alone is sufficient to mediate the tethering or engulfment of apoptotic cells by stabilin-2.
Stabilin-2 is involved in the clearance of aged/apoptotic cells and the production of anti-inflammatory cytokine by HMDMs. To determine whether stabilin-2 is expressed in macrophages, which perform a crucial function in the uptake of apoptotic cells as professional phagocytes, we conducted RT-polymerase chain reaction (PCR) using two different probes and determined that stabilin-2 is expressed in HMDMs, human alveolar macrophages (HAMs), and several macrophage cell lines, including phorbol myristate acetate (PMA)-treated THP-1 and P388D1 cells (Figure 6a). In an effort to assess the functional role of stabilin-2 in a more physiological context, we conducted experiments using HMDMs. In our flow cytometry analyses, we verified that stabilin-2 shRNA, but not control scrambled shRNA, caused a significant reduction in the levels of stabilin-2 expression on the surfaces of HMDMs (Figure 6b). The engulfment of aged RBCs was more pronounced in cases in which HMDMs were utilized as phagocytes. In this case, the percentage of the cells that engulfed aged RBC was approximately $60 \%$, and half of them harbored more than five RBCs. The downregulation of stabilin-2 significantly attenuated the uptake of aged RBCs in $\mathrm{CD}^{+} 8^{+} \mathrm{HMDMs}$ (Figure 6c). The percentage of $\mathrm{CD}^{+} 8^{+} \mathrm{HMDMs}$ that did not engulf RBC after co-culturing with aged RBCs increased from $38.6 \pm 3.9$ to $55.4 \pm 5.5$, and the percentage of the HMDMs engulfing more than five RBCs decreased significantly from $29.9 \pm 3.2$ to $10.4 \pm 2.1$ (Figure $6 \mathrm{~d}$ ). We attempted to determine whether monoclonal antibody against human stabilin-2 (mAb 5G3) inhibits the engulfment of aged RBCs and apoptotic Jurkat $T$ cells. mAb 5 G3 also inhibited the percentage of HMDM engulfing aged RBC (from $59.3 \pm 3.8$ to $44.5 \pm 5.3$ ) (Figure 6e), and the percentage of the HMDMs engulfing 

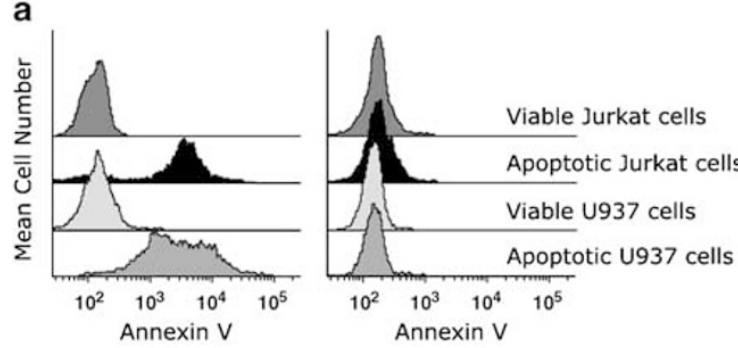

C

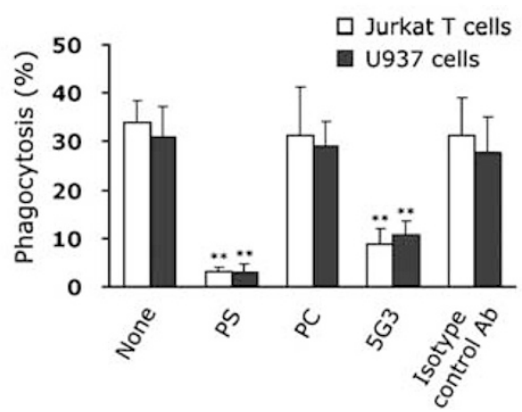

b

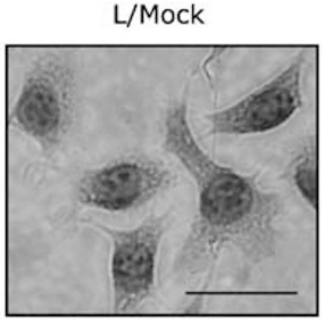

d
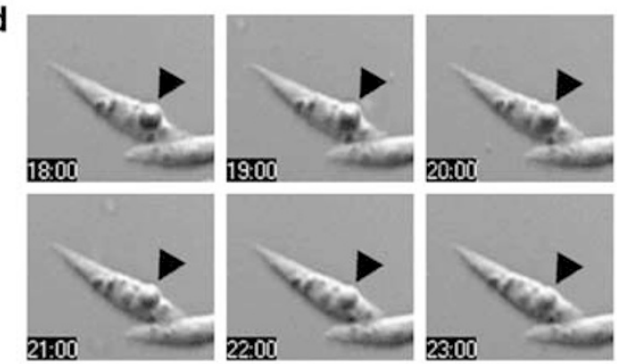

Figure 4 Stabilin-2 mediates the binding and engulfment of apoptotic cells via the recognition of PS. (a) Jurkat T and U937 cells were treated with anti-Fas antibody $(100 \mathrm{ng} / \mathrm{ml}, 6 \mathrm{~h})$ or etoposide $(100 \mathrm{ng} / \mathrm{ml}, 4 \mathrm{~h})$, respectively, to induce apoptosis. Apoptotic cells were stained with PI (right panel) or annexin V (left panel) and analyzed via flow cytometry. (b) Representative images of the engulfment of apoptotic Jurkat T cells in L/Stab-2 and L/Mock cells. Scale bar, $50 \mu \mathrm{m}$. (c) L/Stab-2 cells were preincubated with PS liposomes, PC liposomes, mAb 5G3, or isotype-matched control Ab for $1 \mathrm{~h}$ at $37^{\circ} \mathrm{C}$ before the addition of apoptotic Jurkat $\mathrm{T}(\square)$ or U937 ( $\mathbf{\square}$ ) cells. The percentages of cells engulfing apoptotic cells were determined. The results are expressed as the means \pm S.D. from at least three experiments. ANOVA: $P<0.01$. (d) Individual frames from Supplementary Movie 3, showing the engulfment of apoptotic cells (black arrowheads) by L/Stab-2 cells

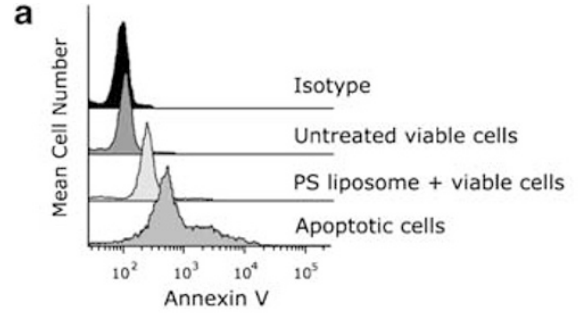

C

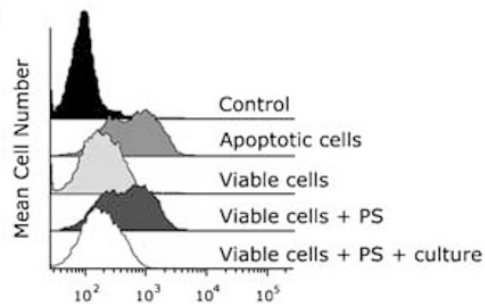

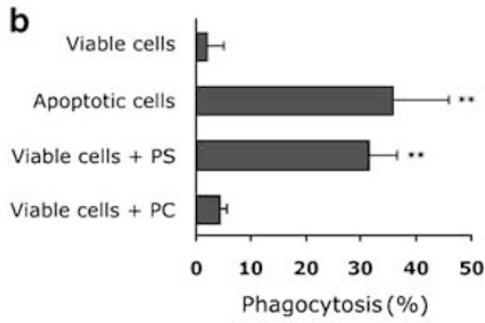

d

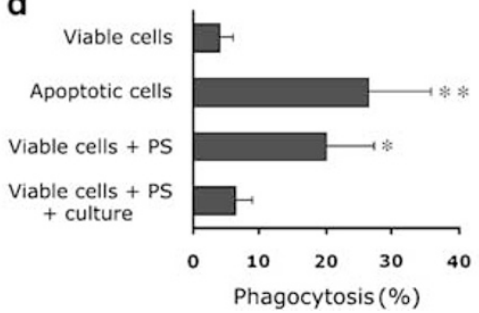

Figure 5 Stabilin-2 expressing cells engulf viable Jurkat T cells that coated PS externally via liposome transfer. (a) Reconstitution of PS on the surfaces of viable Jurkat T cells. PS levels on the surface were analyzed via flow cytometry using annexin V-FITC. A representative result of three independent experiments is shown. (b) Engulfment of viable cells, apoptotic Jurkat T cells, or viable Jurkat T cells coated with liposomes containing brain-derived PS or PC in L/Stab-2 cells. The results are expressed as the means \pm S.D. from at least three experiments. ANOVA: ${ }^{* \star} P<0.01$. (c) Removal of PS on the surfaces of PS-coated viable Jurkat T cells. PS levels on the surfaces were analyzed by flow cytometry using annexin V-FITC. A representative result of three independent experiments is shown. (d) Engulfment of viable cells, apoptotic Jurkat T cells, viable Jurkat T cells coated with liposomes containing brain-derived PS, or viable Jurkat T cells coated with PS liposomes but then washed with bovine serum albumin and cultured for $6 \mathrm{~h}$. The results are expressed as the means \pm S.D. from at least three experiments. ANOVA: ${ }^{*} P<0.01 ;{ }^{*} P<0.05$

more than five RBCs was reduced more efficiently, to the same degree as was observed with stabilin-2 shRNA (data not shown). When apoptotic Jurkat $\mathrm{T}$ cells were used as target cells, the HMDMs engulfed 1-3 apoptotic cells, and the reduction of ingested cells may be the result of the different size of the target cells. The percentage of macrophages that engulfed apoptotic cells was inhibited from $29.4 \pm 4.1$ to $11.8 \pm 1.5$ in the presence of $\mathrm{mAb} 5 \mathrm{G} 3$, similar to that induced by the PS liposome (Figure 6e). However, the blockade of the stabilin-2 receptor with shRNA 
a

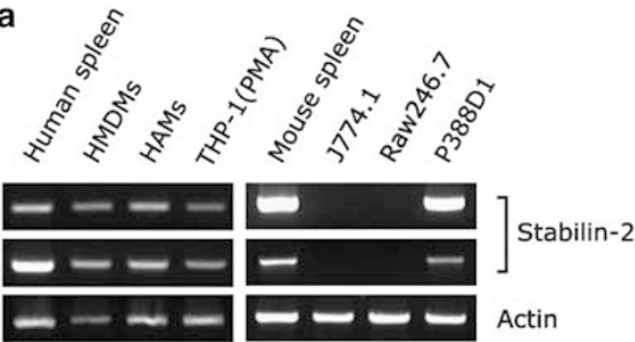

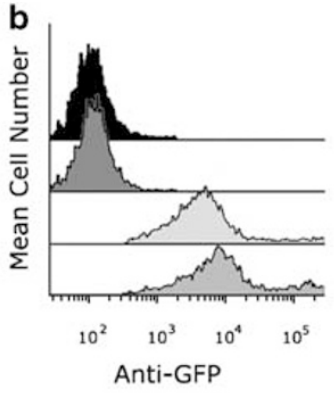

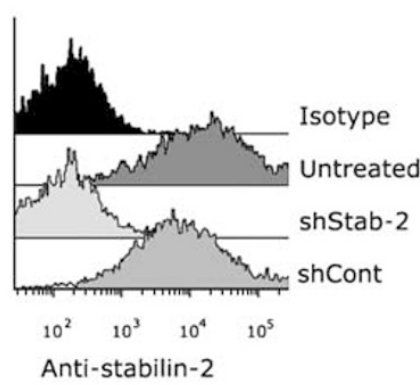

Anti-stabilin-2
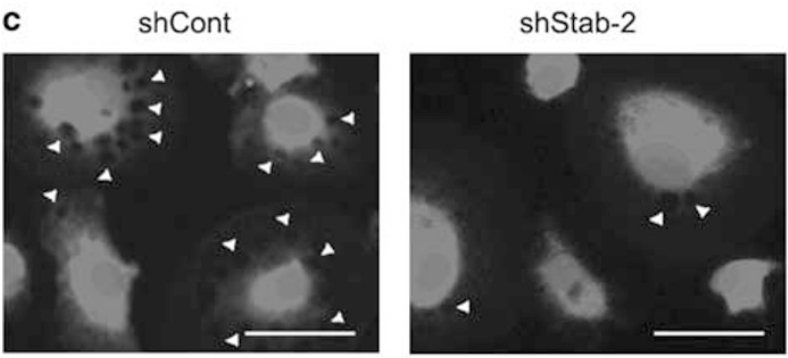

d

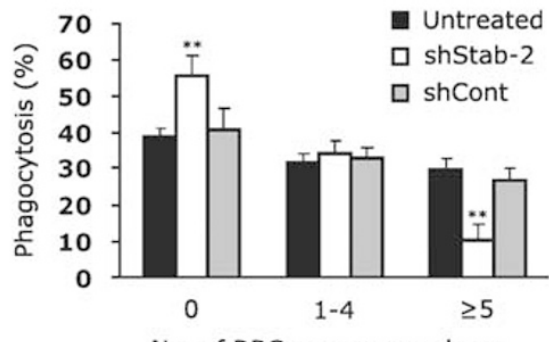

No. of RBCs per macrophage

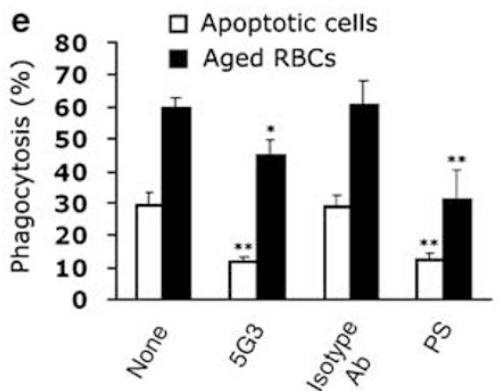

Figure 6 Stabilin -2 is involved in the clearance of aged RBCs and apoptotic cells by HMDMs. (a) Gene transcripts for stabilin-2 were analyzed in several primary macrophages and macrophage cell lines via RT-PCR using two different primers. HMDMs, human monocyte-derived macrophages; HAMs, human alveolar macrophages. (b) Confirmation of shRNA-induced the suppression of stabilin-2 expression in HMDMs. HMDMs were infected with a lentivirus harboring either stabilin-2 shRNA (shStab-2) or scrambled control shRNA (shCont). At $72 \mathrm{~h}$ after infection, the expression of GFP protein (left panel) and the suppression of stabilin-2 expression (right panel) were analyzed via flow cytometry. (c) Representative images of the defective engulfment of aged RBCs by stabilin-2 ${ }^{\mathrm{kd}} \mathrm{HMDM}$. Stabilin-2 $2^{\mathrm{kd}} \mathrm{HMDMs}$ or irrelevant knockdown HMDMs were incubated with aged RBCs, stained with anti-CD68 antibody (red) and DAPI (blue), and observed via confocal microscopy. Scale bar, $25 \mu \mathrm{m}$. (d) Effects of stabilin-2 shRNA in the clearance of aged RBCs. Phagocytosis assays were conducted in Stabilin- ${ }^{\mathrm{kd}} \mathrm{HMDMs}$ or irrelevant knockdown HMDMs, and the percentages of macrophages that carried none, $1-4$, or $\geqslant 5$ aged RBCs were determined. The results are expressed as the means \pm S.D. from at least three experiments. ANOVA: ${ }^{* \star} P<0.01$. (e) Effects of a monoclonal anti-stabilin-2 antibody (5G3) in the clearance of apoptotic cells and aged RBCs. HMDMs were pretreated with mAb $5 \mathrm{G} 3$ or isotype-matched control Ab before the addition of aged RBCs or apoptotic Jurkat T cells. Phagocytosis assays were conducted, and the percentages of macrophages that harbored aged RBCs or apoptotic cells were determined. In the phagocytosis of apoptotic cells, the quenching of fluorescence derived from externally bound cells using trypan blue allowed for the determination of the engulfing HMDMs. The results are expressed as the means \pm S.D. from three independent experiments. ANOVA: ${ }^{\star \star} P<0.01 ;{ }^{*} P<0.05$

or mAb $5 G 3$ induced a partial inhibition of aged and apoptotic cell engulfment. This may be the result of significant redundancy in apoptotic cell recognition, the result of using many macrophage receptors simultaneously. ${ }^{3}$

An important issue with regard to the uptake of apoptotic cells is the consequential resolution of inflammatory responses. $^{2}$ The potential anti-inflammatory effects of the recognition and uptake of apoptotic cells may explain the quiet, non-inflammatory nature of apoptotic cell removal during development and tissue remodelling. As the PSdependent ingestion of apoptotic cells is associated with the production of anti-inflammatory cytokines, ${ }^{26}$ we attempted to evaluate the potential for stabilin-2 to promote the production of the anti-inflammatory cytokine, transforming growth factor$\beta$ (TGF- $\beta$ ), using HMDMs isolated from normal human donors.
As was observed with aged cells and PS-containing liposomes, the pretreatment of the cells with anti-stabilin-2 antibody (5G3) resulted in increased TGF- $\beta$ production (Figure 7a). Similar to what was observed with HMDM, mAb 5 G3 also resulted in increased TGF- $\beta$ production in the L/Stab-2 cells (Figure 7b). Thus, these results provide evidence that stabilin-2 performs an important function in the uptake of aged cells and delivers a 'tickling signal', which modulates the immune response via the secretion of antiinflammatory cytokines.

\section{Discussion}

The externalization of PS that is normally observed within the inner leaflet of the cell membrane occurs during apoptosis, 

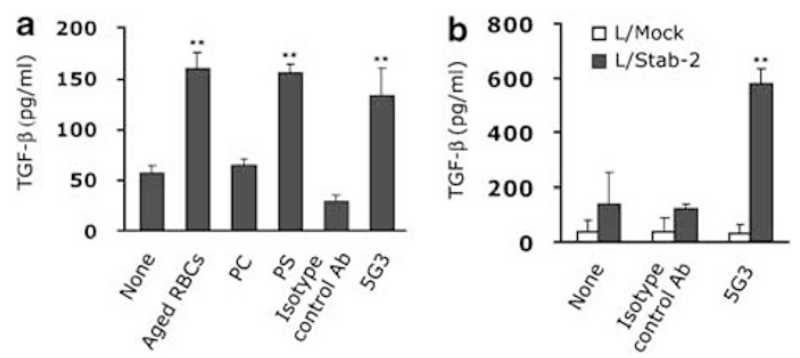

Figure 7 Stabilin-2 activation releases anti-inflammatory cytokine, TGF- $\beta$ in HMDMs (a) and L cell transfectants (b). HMDMs were incubated with aged RBCs, PS liposome, PC liposome, mAb 5G3, or isotype-matched control Ab. L/Stab-2 or L/Mock cells were incubated with $\mathrm{mAb} 5 \mathrm{G} 3$ or isotype-matched control Ab. Supernatants were evaluated for TGF- $\beta$ production by ELISA $18 \mathrm{~h}$ later. The results are expressed as the means \pm S.D. from at least three experiments. ANOVA: ${ }^{* *} P<0.01$

and is the best characterized event that can be used to distinguish live cells from dead ones. ${ }^{27}$ For the rapid and efficient clearance of apoptotic cells, phagocytes must recognize PS on the apoptotic cell surface via either the PS receptor or secreted bridging molecules. Although soluble bridging molecules such as MFG-E8 ${ }^{8}$ have been shown to mediate the PS-dependent engulfment of apoptotic cells, membrane receptors that directly recognize PS remain to be identified. Several membrane receptors have been demonstrated to be involved in the clearance of apoptotic cells, including the PSR, ${ }^{5,28} \mathrm{CD}_{14}{ }^{7}$ and scavenger receptors including CD36. ${ }^{11}$ Among these, the PSR is the most salient representative, as it can recognize $P S$ in a stereospecific manner. ${ }^{5}$ However, recent studies suggest considerable ambiguity regarding the localization of PSR on the cell surface $^{29}$ and whether it is crucial for the clearance of apoptotic cells. ${ }^{30,31}$ The results of previous studies have demonstrated that CD14 and CD36 are involved in the phagocytosis of apoptotic cells as membrane receptors in HMDMs. However, CD14 appears unlikely to bind preferentially to apoptotic-cell-associated PS, and does not function as a PS-receptor on HMDMs. ${ }^{32}$ It remains unclear as to whether CD36 mediates the engulfment of apoptotic cells via direct binding to PS. ${ }^{11}$ The results of the present study suggest that stabilin-2 is a strong candidate for a PS-specific membrane receptor. Four lines of evidence bolster the proposition that stabilin-2 is involved in the clearance of apoptotic cells in human macrophages. First, Stabilin-2-expressing cells were shown to mediate the binding and engulfment of aged and apoptotic cells, and this binding and engulfment activity was inhibited significantly by anti-stabilin-2 antibody or stabilin-2specific shRNA. Second, stabilin-2 recognizes PS in a stereospecific manner. Third, the blockade of stabilin-2 with shRNA or mAb $5 \mathrm{G} 3$ resulted in a reduction in phagocytosis in primary HMDMs. Although the blockade of stabilin-2 receptor partially inhibited the percentage of macrophages engulfing aged RBC, by $\sim 25 \%$, the percentage of the macrophages engulfing more than five aged RBCs was reduced more efficiently, by approximately $65 \%$. Fourth, the introduction of PS into the outer leaflets of viable cells substantially induced their uptake by stabilin-2, thereby suggesting that stabilin-2 may function as a tethering receptor, which potentially binds directly to PS. Thus, our results indicate that stabilin-2 performs an important function as a PS receptor for cell corpse clearance in macrophages.

In addition to PS-recognizing molecules, the engulfment of apoptotic cells requires the coordinated activity of several cellsurface receptors, which ultimately mediate the internalization of bound apoptotic cells. Therefore, two steps, referred to as 'tethering' (recognition of PS) and 'tickling' (internalization of apoptotic cells and activation of downstream signalling pathways) are required for the clearance of apoptotic cells. The evidence collected thus far suggests that a host of membrane receptors, including the vitronectin receptor, CD36, and CD14, function in the tethering of apoptotic cells to phagocytes, and that a tickling signal is generated as the result of interactions between PS and the PS-receptor. ${ }^{33}$ The findings of recent studies have shown that the Mer receptor functions as a tickling receptor. ${ }^{34}$ Does stabilin-2 simply provide tethering signals or does it deliver both signals? The tickling step involves the internalization of tethered cellular corpses and the induction of an active anti-inflammatory response in phagocytes, following binding to the cellular corpse. In this study, we have determined that the blockade of stabilin-2 blocks the interaction between apoptotic cells and phagocytes. Although stabilin-2 expression may have generated the expression of additional 'tickling receptors', the activation of stabilin-2 with the mAb stimulates the production of TGF- $\beta$ in HMDMs and stabilin-2-expressing $L$ cells. Thus, our data indicate that stabilin-2 may aid in the resolution of inflammation by contributing to both the clearance of apoptotic cells and the secretion of anti-inflammatory cytokines, and thus may prove to be responsible for both the tethering and the tickling of phagocytes.

Only few reports have thus far addressed the velocities of apoptotic cell engulfment in phagocytes. Microglial cells phagocytosed an apoptotic cerebellar granule neuron within 1-2 min. $^{35}$ IgG-opsonized erythrocytes have been shown to be ingested more slowly (5-7 min) by Raw264.7 macrophages. ${ }^{36}$ In this study, we determined that aged RBC was ingested quickly, within 2-4s. For the rapid and efficient clearance of apoptotic cells, a single PS receptor with both tethering and tickling functions on the surface of phagocytes is likely required. In this regard, stabilin-2, which is capable of quickly ingesting apoptotic cells, is strongly suggested to be a key molecule for the clearance of apoptotic cells without causing inflammation. However, in our system, apoptotic cell engulfment was delayed for $\sim 5 \mathrm{~min}$. The difference in ingestion times between the aged RBCs and the apoptotic cells may be explained by the sizes of the target cells or by differences in engulfment mechanisms. According to our results, macrophages engulfed many numbers of aged RBCs (3-15 cells per cell) as compared with what was observed with the apoptotic cells (1-3 cells per cell). It remains possible that the engulfment of apoptotic cells may require another complication to the current simple model, in which PS exposed on the cell surface functions as a direct target for recognition by stabilin-2. Recently, it has been suggested that calreticulin may function as a second general recognition ligand, via binding and activating LRP or CD91 on engulfing cells. ${ }^{14}$ Arur et al. ${ }^{37}$ have suggested that annexin I may function in the facilitation of or enhancement of the recognition 
of PS by the receptor. Whether calreticulin or annexin I is related to stabilin-2-mediated phagocytosis is currently unclear, and will require further study.

Unlike other molecules, which are expressed ubiquitously, stabilin-2 is expressed in specific cell types, including sinusoidal endothelial cells of the spleen, lymph node, and liver, ${ }^{21}$ as well as macrophages. It has been reported in previous studies that stabilin-2 is only barely detectable in monocytes/macrophages. ${ }^{20}$ However, we have determined, via RT-PCR, that stabilin-2 is expressed in HMDMs, HAMs, and several macrophage cell lines, including PMA-treated THP-1 and P388D1. Stabilin-2 was also detected in HMDMs via flow cytometry using mAb 5G3, and its expression was specifically downregulated by shRNA against stabilin-2. This discrepancy may be explained by possible differences in antibody epitopes and RT-PCR conditions. In addition, a few studies have demonstrated that stabilin-1, the closest homologue of stabilin-2, is expressed in macrophages. ${ }^{19,21}$ Stabilin-1 and stabilin-2 have similar domain structures and expression patterns, and also operate as scavenger receptors. ${ }^{17,19,20}$ In this point, stabilin-1 may be also involved in the clearance of apoptotic cells in macrophages. It remains to be determined whether two proteins are functionally linked.

In summary, our results demonstrate for the first time that stabilin-2 is a novel PS receptor that provides tethering and tickling signals, and therefore performs a crucial role in the rapid clearance of aged and apoptotic cells and the resolution of inflammation. The identification of stabilin-2 as a receptor that directly recognizes PS on the surfaces of apoptotic cells would facilitate the elucidation of the molecular mechanisms by which apoptotic cells are recognized and phagocytosed in the human body.

\section{Materials and Methods}

Reagents. mAb directed against c-Myc (clone 9E10) was obtained from Santa Cruz. mAb directed against CD68 (clone KP1) was purchased from Abcam. mAb directed against Fas was purchased from Upstate. Alexa Fluor 568 or 647conjugated goat anti-mouse $\lg G$ antibodies were obtained from Molecular Probes. Etoposide was acquired from Sigma. Human and mouse spleen cDNAs were obtained from Invitrogen. All phospholipids were purchased from Avanti Polar Lipids.

Cell culture. Human monocytes were obtained from normal donors, isolated using monocyte isolation kit II systems (Milteny Biotec.) and cultured in X-vivo 10 (BioWhitaker) containing 10\% human serum. The differentiated macrophages (HMDMs) were utilized at 7-10 days of culture. Mouse fibroblast $L$ cells (ATCC CCL-1) were generously donated by Dr M Takeichi (Kyoto University, Japan), and were grown in Dulbecco's modified Eagle medium (DMEM) supplemented with 10\% heat-inactivated FBS and the appropriate antibiotics. U937, a human monocytic cell line, and Jurkat T, a human leukemic cell line, were maintained in RPMI-1640 medium containing $10 \%$ FBS.

Production of $\mathbf{m A b}$. To generate monoclonal anti-human stabilin-2 antibodies, a His-tagged recombinant protein corresponding to amino acids 1173-1727 was expressed in E. coli and purified with Ni-NTA resin, in accordance with the manufacturer's instructions. Six mice were immunized with $20 \mu \mathrm{g}$ of purified recombinant protein each, at 2-week intervals. All mAb production was performed by Dinona Inc. (Seoul, Korea). Supernatants resulting from the fusion were tested by flow cytometry for $L$ cells transfected with stabilin-2 cDNA or empty vector. One hybridoma supernatant (clone 5G3, IgG1, lambda chain) was selected. This mAb (5G3) was shown to be reactive via flow cytometry and immunohistochemical staining, and did not crossreact with orthologous proteins including mouse and rat stabilin-2, nor with paralogous proteins such as human stabilin-1 (Supplementary

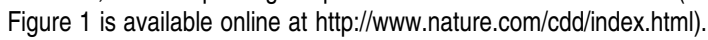

Transfection. The complete stabilin-2 cDNA was amplified from human spleen cDNA (Invitrogen) and cloned into pcDNA3.1(-)/Myc-His vector (Invitrogen). Mouse fibroblast $L$ cells were transfected with human stabilin-2 cDNA using Lipofectamine (Invitrogen). For stable transfection, L cells were selected in G418 ( $400 \mu \mathrm{g} / \mathrm{ml})$. Individual G418-resistant colonies were isolated after 10-12 days of culture. The final clones were designated L/Stab-2-\#. Negative control clones were selected randomly from transfection with empty vector (L/Mock). The expression of stabilin-2 was assessed via Western blotting and flow cytometry.

Fluorescence-activated cell sorting analysis. For the analysis of surface staining, the cells were incubated with $1 \mu \mathrm{g} / \mathrm{ml}$ of mAb $5 \mathrm{G} 3$ on ice for $30 \mathrm{~min}$ with gentle agitation every $5 \mathrm{~min}$. The cells were washed twice, resuspended in PBS, and incubated with $4 \mu \mathrm{g} / \mathrm{ml}$ of FITC-conjugated goat anti-mouse lgG antibody for 45 min on ice. After three washes in PBS, the cells were resuspended in PBS, and analyzed via flow cytometry on a FACScalibur cytometer (BD Biosciences).

Liposome preparation and liposome incorporation assay. Liposomes containing the indicated phospholipid and PC at a molar ratio of $50: 50$ were prepared. The lipids were mixed in chloroform and dried under nitrogen gas. The dried lipids were then resuspended in PBS at a final concentration of $5 \mathrm{mM}$. The mixture was mixed, and then sonicated for $10 \mathrm{~min}$ on ice. Fluorescence-labelled liposomes were prepared in the presence of $N$-(lissamin rhodamine B sulfonyl)-L- $\alpha$-phosphatidylethanolamine (Avanti Polar Lipids) at $1 \%$ of total phospholipids, as described previously. ${ }^{38}$ The affinity of fluorescent liposomes to $L$ cell transfectants was quantified with a fluorescent microplate reader (Biolumin 960, Molecular Dynamics) set to an excitation wavelength of $549 \mathrm{~nm}$ and an emission wavelength of $565 \mathrm{~nm}$.

Reconstitution of plasma membrane outer leaflet with PS. The association of liposomes containing PS with Jurkat T cells was conducted as previously described ${ }^{39}$ with slight modifications. In brief, the cells were collected, washed twice, and treated with $10 \mu \mathrm{M} \mathrm{N}$-ethylmaleimide (Sigma) for $5 \mathrm{~min}$ at $37^{\circ} \mathrm{C}$ in order to inhibit aminophospholipid translocase (APT) activity. The cells were then exposed to varying quantities of liposomes for $30 \mathrm{~min}$ at $37^{\circ} \mathrm{C}$, washed twice in PBS, and used immediately thereafter. Surface PS was verified via evaluation of annexin V-FITC binding ability, as determined via flow cytometry. In some cases, the added PS was removed by washing the cells three times in PBS containing $2 \%$ bovine serum albumin, and the cells were incubated for $6 \mathrm{~h}$ in tissue culture medium at $37^{\circ} \mathrm{C}$ in order to allow for the translocation of remaining PS to the inner leaflet via APT. PS removal was also verified via flow cytometry, as described above.

Binding and phagocytosis of aged RBCs. Aged RBCs were prepared via the incubation of cells in PBS $\left(20 \%\right.$ hematocrit) at $37^{\circ} \mathrm{C}$, as described previously. ${ }^{10}$ Exposure of PS on the surface of aged RBCs was detected via annexin V-FITC using an annexin V Apoptosis detection kit (Santa Cruz). Aged RBCs were added to the $L$ cell transfectants or HMDMs, and incubated for $1 \mathrm{~h}$ to assess binding in the presence or absence of $\mathrm{mAb}(5 \mathrm{G} 3)(20 \mu \mathrm{g} / \mathrm{ml})$, isotypematched control $\mathrm{Ab}(\mathrm{lgG1}, 20 \mu \mathrm{g} / \mathrm{ml})$, or $10 \mu \mathrm{M}$ liposome containing PC, or $50: 50$ molar ratios of PS:PC, PI:PC, PG:PC, PE:PC, PA:PC, or PG:PC. After washing away the unbound RBCs, the uningested RBCs were lysed via the addition of deionized $\mathrm{H}_{2} \mathrm{O}$ for $10 \mathrm{~s}$, followed by immediate replacement with DMEM as described. ${ }^{13}$ The cells were fixed with methanol and stained with a Diff Quick staining kit (IMEB Inc.). The binding and phagocytosis of aged RBCs were quantified via light microscopy before and following hypotonic lysis, respectively. The percentages of binding and phagocytosis were determined as the percentages of phagocytes positive for binding and engulfment, respectively. At least 100 cells were scored per well, and the experiments were repeated at least three times.

In certain experiments, the phagocytosis assay was conducted in the presence of monoclonal anti-stabilin-2 antibody. L cell transfectants or HMDMs were preincubated with anti-stabilin-2 antibody for $1 \mathrm{~h}$ at $37^{\circ} \mathrm{C}$ before the addition of aged RBCs.

Phagocytosis of apoptotic cells. Apoptotic cells were added to the $L$ cell transfectants or HMDMs, and incubated for $1 \mathrm{~h}$ to evaluate uptake in the presence of mAb $5 \mathrm{G} 3(20 \mu \mathrm{g} / \mathrm{ml})$, isotype-matched control Ab (lgG1, $20 \mu \mathrm{g} / \mathrm{ml})$, or $10 \mu \mathrm{M}$ liposome containing PS or PC. After extensive washing away the uningested cells, 
the cells were stained using a Diff Quick staining kit, and the uptake of apoptotic cells was quantified under a light microscope. Under a light microscope, the engulfed apoptotic cells were lightly stained, and the nuclei of engulfing cells were dislocated by the engulfed apoptotic cells. Cells harboring lightly stained apoptotic cells and manifesting nuclear dislocation by apoptotic cells were scored as engulfing cells. This convention may have underestimated the number of engulfing cells. The percentage of phagocytosis was determined as the percentage of phagocytes positive for engulfment.

In the phagocytosis assay using HMDMs, apoptotic Jurkat T cells were labeled with PC-NBD and added to HMDMs. After $1 \mathrm{~h}$ of incubation, the uningested apoptotic cells were removed by extensive washing. To further identify the engulfed cells, fluorescence derived from bound apoptotic cells (remnant cells) was quenched using the trypan blue described previously. ${ }^{40}$ HMDMs engulfing fluorescent-labelled apoptotic cells were quantified under confocal microscopy.

Time-lapse video microscopy. L/Stab-2 cells were seeded into collagen type I coated dishes (IWAKI, Asahi techno glass). The culture dishes were placed in a chamber on the microscope (Leica) at $37^{\circ} \mathrm{C}$ in an atmosphere of $5 \% \mathrm{CO}_{2}$. DIC (differential interference contrast) images were acquired using a $\times 100$ objective and a cooled CCD camera (Cool Snap HQ, Photometrics). Images were controlled using Metamorph image processing software (Universal Imaging Corp.). Two hours after the addition of aged RBCs or apoptotic cells, we began to collect images every $2-20 \mathrm{~s}$ for $2 \mathrm{~h}$.

Lentivirus-mediated shRNA production. Three 20-nt shRNA sequences, shRNA1: 5'-CAAACTGGAATGCAAATGCC-3', shRNA2: 5'-TGGCAA GGAAGGCTGACCTC-3', and shRNA3: 5'-CGCAGCAGTGGAATTGTCAT-3', corresponding to bases $840-959,1324-1343$, and 3174-3193, respectively, in the stabilin-2 mRNA sequence (accession number: NM_017564) were designed. Candidate oligonucleotides were synthesized and cloned into the pSuper/basic vector (OligoEngine). pSuper/shRNA2 significantly downregulated stabilin-2 protein expression in the CHO-K1 cells co-transfected with a vector expressing stabilin-2, and the expression cassette of pSuper/shRNA2 was subcloned into the pRNATU6.1/Lenti lentiviral vector (GenScript). Scrambled shRNA (shCont) was employed as a control for stabilin-2 shRNA (shStab-2). All constructs were confirmed via sequence analysis. Lentiviral transduction was conducted in accordance with the manufacturer's instructions. In brief, the pRNAT-shStab-2 vector was cotransfected with the packaging vector (Invitrogen) into 293FT cells (Invitrogen). The supernatant was collected after $72 \mathrm{~h}$, and filtered through a $0.22 \mu \mathrm{m}$ pore acetate filter. HMDMs were seeded at $2 \times 10^{5} \mathrm{cell} / \mathrm{ml}$ into 24 -well plates. Lentiviral particles and $8 \mu \mathrm{g} / \mathrm{ml}$ of polybrene (Sigma) were added to the culture. In our system, using lentivirusmediated-shRNA (shStab-2), the depletion of stabilin-2 via shRNA-mediated gene silencing was determined to be effective 3 days after infection. pRNAT-shStab-2 and pRNAT-shCont vectors also harbored the transgene for GFP, allowing for the monitoring of infection efficiency.

RNA isolation, reverse transcription and PCR. Total RNA was isolated from spleen tissues, as well as several primary macrophages and macrophage cell lines, using standard Trizol RNA isolation protocol (Invitrogen). Reverse transcription was performed with M-MLV reverse transcriptase (Promega) using $2 \mu \mathrm{g}$ of total RNA (Invitrogen) for $50 \mathrm{~min}$ at $42^{\circ} \mathrm{C}$, followed by $3 \mathrm{~min}$ at $95^{\circ} \mathrm{C}$. The human stabilin-2 was amplified in a Perkin-Elmer 2400 PCR thermocycler under the following conditions: $94^{\circ} \mathrm{C}$ for $2 \mathrm{~min}$ (initial denaturation); $94^{\circ} \mathrm{C}$ for $30 \mathrm{~s}$, $58^{\circ} \mathrm{C}$ for $30 \mathrm{~s}, 72^{\circ} \mathrm{C}$ for $45 \mathrm{~s}$ ( $35 \mathrm{cycles}$ ); and $72^{\circ} \mathrm{C}$ for $10 \mathrm{~min}$ (final extension). The primers utilized in this study are shown in Supplementary Table 1.

TGF- $\beta$ assays. HMDMs were treated with either aged RBCs, $10 \mu \mathrm{M}$ liposomes containing $50 \mathrm{molar} \% \mathrm{PS}$ or PC, mAb $5 \mathrm{G} 3(20 \mu \mathrm{g} / \mathrm{ml})$, or isotype-matched control $\mathrm{Ab}(20 \mu \mathrm{g} / \mathrm{ml})$. The supernatants were evaluated for cytokine production via ELISA $18 \mathrm{~h}$ later (R\&D system). $\mathrm{L}$ cell transfectants were treated with $\mathrm{mAb} 5 \mathrm{G} 3$ or isotypematched control $A b$ and assessed for TGF- $\beta$ production $18 \mathrm{~h}$ later.

Statistical analysis. Statistical significance was assessed via ANOVA. A $P$-value of $<0.05$ was considered to be statistically significant.

Acknowledgements. This work was supported by a National Research Laboratory Program (M10104000036-01J0000-01610); by the Grant No. RTI04-0101 from the Regional Technology Innovation Program of the MOCIE, Advanced
Medical Technology Cluster for Diagnosis and Prediction at Kyungpook National University from MOCIE; and by the Brain Korea 21 Project, in 2007.

1. Strasser A, O'Connor L, Dixit VM. Apoptosis signaling. Annu Rev Biochem 2000; 69 : 217-245.

2. Savill J, Fadok V. Corpse clearance defines the meaning of cell death. Nature 2000; 407: 784-788.

3. Lauber K, Blumenthal SG, Waibel M, Wesselborg S. Clearance of apoptotic cells: getting rid of the corpses. Mol Cell 2004; 14: 277-287.

4. Henson PM, Bratton DL, Fadok VA. Apoptotic cell removal. Curr Biol 2001; 11: R795R805.

5. Fadok VA, Bratton DL, Rose DM, Pearson A, Ezekewitz RA, Henson PM. A receptor for phosphatidylserine-specific clearance of apoptotic cells. Nature 2000; 405: 85-90.

6. Zhou Z, Hartwieg E, Horvitz HR. CED-1 is a transmembrane receptor that mediates cell corpse engulfment in C. elegans. Cell 2001; 104: 43-56.

7. Devitt A, Moffatt OD, Raykundalia C, Capra JD, Simmons DL, Gregory CD. Human CD14 mediates recognition and phagocytosis of apoptotic cells. Nature 1998; 392: 505-509.

8. Hanayama R, Tanaka M, Miwa K, Shinohara A, Iwamatsu A, Nagata S. Identification of a factor that links apoptotic cells to phagocytes. Nature 2002; 417: 182-187.

9. Scott RS, McMahon EJ, Pop SM, Reap EA, Caricchio R, Cohen PL et al. Phagocytosis and clearance of apoptotic cells is mediated by MER. Nature 2001; 411: 207-211.

10. Oka K, Sawamura T, Kikuta K, Itokawa S, Kume N, Kita T et al. Lectin-like oxidized lowdensity lipoprotein receptor 1 mediates phagocytosis of aged/apoptotic cells in endothelial cells. Proc Natl Acad Sci USA 1998; 95: 9535-9540.

11. Fadok VA, Warner ML, Bratton DL, Henson PM. CD36 is required for phagocytosis of apoptotic cells by human macrophages that use either a phosphatidylserine receptor or the vitronectin receptor (alpha v beta 3). J Immunol 1998; 161: 6250-6257.

12. Franc NC, Heitzler $P$, Ezekowitz RA, White K. Requirement for croquemort in phagocytosis of apoptotic cells in Drosophila. Science 1999; 284: 1991-1994.

13. Ogden CA, deCathelineau A, Hoffmann PR, Bratton D, Ghebrehiwet B, Fadok VA et al. $\mathrm{C} 1 \mathrm{q}$ and mannose binding lectin engagement of cell surface calreticulin and CD91 initiates macropinocytosis and uptake of apoptotic cells. J Exp Med 2001; 194: 781-795.

14. Gardai SJ, McPhillips KA, Frasch SC, Janssen WJ, Starefeldt A, Murphy-Ullrich JE et al. Cell-surface calreticulin initiates clearance of viable or apoptotic cells through transactivation of LRP on the phagocyte. Cell 2005; 123: 321-334.

15. Platt N, da Silva RP, Gordon S. Recognizing death: the phagocytosis of apoptotic cells. Trends Cell Biol 1998; 8: 365-372.

16. Gardai SJ, Bratton DL, Ogden CA, Henson PM. Recognition ligands on apoptotic cells: a perspective. J Leukoc Biol 2006; 79: 896-903.

17. Politz $O$, Gratchev A, McCourt PA, Schledzewski K, Guillot $P$, Johansson $S$ et al. Stabilin-1 and -2 constitute a novel family of fasciclin-like hyaluronan receptor homologues. Biochem J 2002; 362 (Part 1): 155-164.

18. Zhou B, Weigel JA, Fauss L, Weigel PH. Identification of the hyaluronan receptor for endocytosis (HARE). J Biol Chem 2000; 275: 37733-37741.

19. Adachi $\mathrm{H}$, Tsujimoto M. FEEL-1, a novel scavenger receptor with in vitro bacteria-binding and angiogenesis-modulating activities. J Biol Chem 2002; 277: 34264-34270.

20. Tamura Y, Adachi H, Osuga J, Ohashi K, Yahagi N, Sekiya M et al. FEEL-1 and FEEL-2 are endocytic receptors for advanced glycation end products. J Biol Chem 2003; 278: 12613-12617.

21. Falkowski M, Schledzewski K, Hansen B, Goerdt S. Expression of stabilin-2, a novel fasciclin-like hyaluronan receptor protein, in murine sinusoidal endothelia, avascular tissues, and at solid/liquid interfaces. Histochem Cell Biol 2003; 120: 361-369.

22. Nose A, Nagafuchi A, Takeichi M. Expressed recombinant cadherins mediate cell sorting in model systems. Cell 1988; 54: 993-1001.

23. Gigli I, Nelson Jr RA. Complement dependent immune phagocytosis. I. Requirements for $C^{\prime} 1, C^{\prime} 4, C^{\prime} 2, C^{\prime} 3$. Exp Cell Res 1968; 51: 45-67.

24. Fadok VA, Voelker DR, Campbell PA, Cohen JJ, Bratton DL, Henson PM. Exposure of phosphatidylserine on the surface of apoptotic lymphocytes triggers specific recognition and removal by macrophages. J Immunol 1992; 148: 2207-2216.

25. Martin SJ, Reutelingsperger CP, McGahon AJ, Rader JA, van Schie RC, LaFace DM et al. Early redistribution of plasma membrane phosphatidylserine is a general feature of apoptosis regardless of the initiating stimulus: inhibition by overexpression of $\mathrm{Bcl}-2$ and $\mathrm{Abl}$. J Exp Med 1995; 182: 1545-1556.

26. Huynh ML, Fadok VA, Henson PM. Phosphatidylserine-dependent ingestion of apoptotic cells promotes TGF-beta1 secretion and the resolution of inflammation. J Clin Invest 2002; 109: 41-50.

27. Schlegel RA, Williamson P. Phosphatidylserine, a death knell. Cell Death Differ 2001; 8: 551-563.

28. Li MO, Sarkisian MR, Mehal WZ, Rakic P, Flavell RA. Phosphatidylserine receptor is required for clearance of apoptotic cells. Science 2003; 302: 1560-1563.

29. Cui P, Qin B, Liu N, Pan G, Pei D. Nuclear localization of the phosphatidylserine receptor protein via multiple nuclear localization signals. Exp Cell Res 2004; 293: 154-163.

30. Bose J, Gruber AD, Helming L, Schiebe S, Wegener I, Hafner M et al. The phosphatidylserine receptor has essential functions during embryogenesis but not in apoptotic cell removal. J Biol 2004; 3: 15. 
31. Mitchell JE, Cvetanovic M, Tibrewal N, Patel V, Colamonici OR, Li MO et al. The presumptive phosphatidylserine receptor is dispensable for innate anti-inflammatory recognition and clearance of apoptotic cells. J Biol Chem 2005; 281: 5718-5725.

32. Devitt A, Pierce S, Oldreive C, Shingler WH, Gregory CD. CD14-dependent clearance of apoptotic cells by human macrophages: the role of phosphatidylserine. Cell Death Differ 2003: 10: 371-382.

33. Hoffmann PR, deCathelineau AM, Ogden CA, Leverrier Y, Bratton DL, Daleke DL et al. Phosphatidylserine (PS) induces PS receptor-mediated macropinocytosis and promotes clearance of apoptotic cells. J Cell Biol 2001; 155: 649-659.

34. Wu Y, Tibrewal N, Birge RB. Phosphatidylserine recognition by phagocytes: a view to a kill. Trends Cell Biol 2006; 16: 189-197.

35. Parnaik R, Raff MC, Scholes J. Differences between the clearance of apoptotic cells by professional and non-professional phagocytes. Curr Biol 2000; 10 : $857-860$.

Supplementary Information accompanies the paper on Cell Death and Differentiation website (http://www.nature.com/cdd)
36. Henry RM, Hoppe AD, Joshi N, Swanson JA. The uniformity of phagosome maturation in macrophages. J Cell Biol 2004; 164: 185-194.

37. Arur S, Uche UE, Rezaul K, Fong M, Scranton V, Cowan AE et al. Annexin I is an endogenous ligand that mediates apoptotic cell engulfment. Dev Cell 2003; 4: 587-598.

38. Kawasaki Y, Nakagawa A, Nagaosa K, Shiratsuchi A, Nakanishi Y. Phosphatidylserine binding of class B scavenger receptor type I, a phagocytosis receptor of testicular sertoli cells. J Biol Chem 2002; 277: 27559-27566.

39. Fadok VA, de Cathelineau A, Daleke DL, Henson PM, Bratton DL. Loss of phospholipid asymmetry and surface exposure of phosphatidylserine is required for phagocytosis of apoptotic cells by macrophages and fibroblasts. J Biol Chem 2001; 276: 1071-1077.

40. Finnemann SC, Rodriguez-Boulan E. Macrophage and retinal pigment epithelium phagocytosis: apoptotic cells and photoreceptors compete for alphavbeta3 and alphavbeta5 integrins, and protein kinase $C$ regulates alphavbeta5 binding and cytoskeletal linkage. J Exp Med 1999; 190: 861-874. 